\title{
High prevalence of osteoporosis in patients with gastric adenocarcinoma following gastrectomy
}

\author{
Jung Sub Lim, Sang Bum Kim, Ho-Yoon Bang, Gi Jeong Cheon, Jong-Inn Lee
}

Jung Sub Lim, Department of Pediatrics, Korea Cancer Center Hospital, Nowon-gu, Seoul 139-706, Korea

Sang Bum Kim, Ho-Yoon Bang, Jong-Inn Lee, Department of Surgery, Korea Cancer Center Hospital, Nowon-gu, Seoul 139-706, Korea

Gi Jeong Cheon, Department of Nuclear Medicine, Korea Cancer Center Hospital, Nowon-gu, Seoul 139-706, Korea

Supported by the Korea Institute of Radiological and Medical Sciences Clinical Research Fund and Korea Science and Engineering Foundation

Correspondence to: Jong-Inn Lee, MD, Department of Surgery, Korea Cancer Center Hospital, Gongneung-dong 215, Nowon-gu, Seoul 139-706, Korea. jilee@kcch.re.kr

Telephone: +82-2-9701217 Fax: +82-2-9782005

Received: August 2, 2007 Revised: October 7, 2007

\begin{abstract}
AIM: To evaluate the prevalence and predictive factors of osteoporosis in patients with gastric adenocarcinoma after gastrectomy.
\end{abstract}

METHODS: The study included 133 patients diagnosed with gastric adenocarcinoma but who did not undergo prior diagnostic work-up for osteoporosis. Bone mineral density (BMD) was measured by dual-energy X-ray absorptiometry (DXA) and vertebral deformity was assessed by plain $X$-rays. We evaluated the effects of age, sex, body mass index (BMI), anemia, back pain, vertebral deformity, tumor staging, reconstruction type, and past medical history to determine predictive factors of osteoporosis in these patients.

RESULTS: The prevalence of osteoporosis in the lumbar spine was $38.3 \%$ (male, $28.9 \%$; female, $54.0 \%$ ), and $15.0 \%$ in the femoral neck (male, $10.8 \%$; female, $22.0 \%$ ). The vertebral deformity rate was $46.6 \%$ (male, $43.4 \%$; female, $52.0 \%$ ). Age, BMI and hemoglobin correlated with BMD $(P<0.01)$. In males, anemia and age $>64$ years were independent predictive factors of osteoporosis in multivariate analysis. In females, back pain was an independent factor for osteoporosis.

CONCLUSION: The results of this study revealed that prevalence of osteoporosis and vertebral bone deformity rate were high in gastric cancer patients, regardless of post-gastrectomy duration and operation type. Early diagnosis and a proper management plan must be established in these patients.

(c) 2007 WJG. All rights reserved.
Key words: Gastric adenocarcinoma; Gastrectomy; Bone mineral density; Osteoporosis; Vertebral deformity; Anemia; Back pain

Lim JS, Kim SB, Bang HY, Cheon GJ, Lee JI. High prevalence of osteoporosis in patients with gastric adenocarcinoma following gastrectomy. World J Gastroenterol 2007; 13(48): 6492-6497

http://www.wjgnet.com/1007-9327/13/6492.asp

\section{INTRODUCTION}

Gastric cancer is one of the most common cancers in the world. Although decreasing in prevalence, it is still the second most common cause of cancer death in the world. The incidence is still very high in East Asia ${ }^{[1]}$. Although the overall prognosis of gastric cancer is poor, the 5-year survival rate for those who receive operative treatment is $55.6 \%$ in Korea. Due to early diagnosis and aggressive surgical intervention, the survival rate is $>90 \%$ in cases of early gastric cancer in Korea ${ }^{[2]}$. However, many surviving patients suffer from the sequelae caused by the surgical procedure. Recently, a 63-year-old woman, who underwent gastrectomy for gastric malignancy, spontaneously developed multiple fractures. It was speculated that the synergistic effects of gastrectomy and other osteoporosis risk factors led to multiple fractures ${ }^{[3]}$. Weight loss, malnutrition, anemia and osteoporosis are common after gastrectomy ${ }^{[4]}$. Following gastric resection, reduced cortical and trabecular bone mass have been reported, however, the mechanism remains unclear ${ }^{[5,6]}$.

The american Gastroenterological Association (AGA) has recommended dual-energy X-ray absorptiometry (DXA) in patients who are alive at least 10 years after gastrectomy ${ }^{[6]}$, based on many reports of post-gastrectomy bone disease $e^{[7-20]}$. Most studies have dealt with patients with peptic ulcer disease, and those focusing on gastric cancer patients are rare. Recently, most gastrectomies have been done in patients with gastric malignancy. However, the age at operation and operation type might differ between peptic ulcer and gastric cancer. The duration after gastrectomy to onset of osteoporosis may also be different. Among the population aged $>50$ years old, gastric cancer detection and operation have recently increased in Korea ${ }^{[2]}$.

In this study, we evaluated the prevalence of osteoporosis and vertebral bone deformity rate in patients 
with gastric adenocarcinoma after gastrectomy. We evaluated the impact of tumor staging, operation type, and other risk factors of osteoporosis. We also evaluated the association between clinical manifestations and osteoporosis for early diagnosis of osteoporosis in gastric cancer patients.

\section{MATERIALS AND METHODS}

One hundred and thirty-three patients with gastric cancer seen between January and December 2006 at the Korea Cancer Center Hospital were included in this study. The criteria for entry in this study were gastric cancer patients: (1) diagnosed with adenocarcinoma; (2) treated with curative gastrectomy and followed up without recurrence; (3) aged > 50 years; (4) never previously diagnosed with osteoporosis, and who had not taken any medication, such as estrogen, bisphosphonate, corticosteroid, and vitamin D; (5) with no other disease that affected bone mineral density (BMD) (like alcoholism, renal failure, chronic liver disease, and immobility); and (6) who could take care of themselves and had an average level of physical activity. Patients' characteristics are described in Table 1. Eightyfour enrolled patients were men and 55 were women, ranging in age from 50 to 80 years (median, 63.9 years).

The authors interviewed all patients closely regarding the known risk factors of osteoporosis, such as family history of osteoporosis, previous fracture, type 2 diabetes, and other chronic diseases and medication states. Anthropometric data (height, weight and BMI), operation record (tumor stage, gastrectomy and reconstruction type), and pathology report were also closely reviewed.

BMD of the lumbar spine (L1-4), and four sites in the proximal femur (femoral neck, Ward's triangle, greater trochanter, and total hip) were measured by DXA (GE Prodigy; Lunar Radiation, Madison, WI, USA). The results of BMD were expressed as absolute values $\left(\mathrm{g} / \mathrm{cm}^{2}\right)$, $\mathrm{T}$ scores (compared with young adults) and $\mathrm{Z}$ scores (compared with age-matched controls) were according to Korean reference values provided by the GE-Lunar database. Osteoporosis was defined as a $\mathrm{T}$ score of $<-2.5$ and osteopenia as a $\mathrm{T}$ score of -1.0 to $-2.5 \mathrm{SD}^{[21]}$. Lateral radiographs of the thoraco-lumbar spine (T-L spine) were evaluated to assess vertebral bone deformity. Vertebral bone deformity was defined when Genant semiquantitative grading was higher than grade $1^{[22]}$. Low BMI was defined as $<18.5 \mathrm{~kg} / \mathrm{m}^{2}$. Anemia was defined as hemoglobin $<11 \mathrm{~g} / \mathrm{dL}$ in women and $<13 \mathrm{~g} / \mathrm{dL}$ in men. High serum alkaline phosphatase (sALP) was defined as $>127 \mathrm{U} / \mathrm{L}$ (normal limit; < $126 \mathrm{U} / \mathrm{L}$ ). Serum albumin, AST/ALT and blood urea nitrogen (BUN)/creatinine were used to rule out severe malnutrition, and liver and renal disease.

\section{Statistical analysis}

Numbers and values were expressed as means \pm SD. Mean quantitative values were compared by Student's $t$ test. Differences in proportions among the groups were analyzed by the $\chi^{2}$ test. Variables relevant to osteoporosis were assessed by univariate analysis: age $(<64 v s \geqslant 64$ years); sex (male vs female); low BMI $\left(<18.5 \mathrm{~kg} / \mathrm{m}^{2}\right)$; tumor stage; gastrectomy and reconstruction type (subtotal gastrectomy with Billroth I and Billroth II reconstruction vs total gastrectomy with Roux-en-Y reconstruction); serum ALP level $(<127$ vs $\geqslant 127 \mathrm{U} / \mathrm{L})$; hemoglobin level (male, $<13 v s \geqslant 13 \mathrm{~g} / \mathrm{dL}$; female, $<11 v \mathrm{~s} \geqslant 11 \mathrm{~g} / \mathrm{dL}$ ); family history of osteoporosis; previous fracture; type 2 diabetes; bone pain; and vertebral deformity. Variables significant by univariate analysis $(P<0.05)$ were included in a multivariate regression analysis. Data analysis was performed with SPSS version 13.0 (Chicago, IL, USA). $P<0.05$ was considered as statistically significant.

\section{RESULTS}

\section{DXA and vertebral deformity}

BMD distribution was different between male and female patients; therefore, we listed BMD according to sex in Table 2. The Z score of L1-4 BMD was decreased -0.55 \pm 1.55 in male and $-0.72 \pm 1.49$ in female patients. However, the $Z$ score of the femur neck was $0.44 \pm 0.97$ in male and $0.74 \pm 1.14$ in female patients. The L1-4 BMD Z score was significantly decreased compared with the femur neck BMD Z score $(P<0.01)$.

Fifty-one $(38.3 \%)$ patients had osteoporosis and 42 $(31.6 \%)$ had osteopenia in lumbar spine BMD, while $20(15.0 \%)$ patients had osteoporosis and $42(31.6 \%)$ had osteopenia in femur neck BMD (Figure 1). The osteoporosis rate was $28.9 \%$ in male and $54.0 \%$ in female patients in lumbar spine BMD. Osteoporosis rate was $10.8 \%$ in male and $22.0 \%$ in female patients in femur neck BMD. The BMI change after gastrectomy $(-2.0 \pm 2.4$, male $v s-2.9 \pm 2.4$ female), anemia rate ( $24 \%$ male $v$ s $58 \%$ female), and TNM stage differed between male and female patients (Table 1). Vertebral bone deformity rate was $43.4 \%$ in male and $52.0 \%$ in female patients.

There was no difference in osteoporosis rate at the lumbar spine $(39.7 \%$ vs $37.3 \%)$ and that of femur neck $(17.2 \%$ vs $13.3 \%)$ between early and advanced gastric cancer. There was no difference in BMD and osteoporosis rate among patients who underwent partial gastrectomy with Billroth I $(40.4 \%)$ or Billroth II reconstruction (38.9\%), and total gastrectomy with Roux-en Y reconstruction $(34.4 \%)$ (Figure 2 ).

\section{Factors for osteoporosis in gastric adenocarcinoma}

With the exception of sex, three variables were correlated with lumbar spine BMD. The correlation coefficients of age, BMI and hemoglobin were $-0.391,0.415$ and 0.274 , respectively $(P<0.01)$. In multiple regression analysis, BMI and hemoglobin level were correlated with BMD in male patients $(P<0.05)$. Age was not a significant factor $(P=0.13)$. On the contrary, BMI and age were significant factors in female patients $(P<0.01)$.

In univariate analysis, six variables were related to osteoporosis (Table 3). Of these factors, age $>64$ years, female sex, BMI $<18.5 \mathrm{~kg} / \mathrm{m}^{2}$, anemia, back pain, and vertebral deformity were significant predictors of osteoporosis. Multivariate analysis disclosed three independent predictors after stratification by sex: serum hemoglobin level $\leqslant 13$ vs $>13 \mathrm{~g} / \mathrm{dL}$ (odds ratio, 4.887; 95\% CI, 1.404-17.011; $P=0.011)$, age $>64$ vs $<64$ years 
Table 1 Patient characteristics (mean \pm SD)

\begin{tabular}{|c|c|c|c|c|c|c|}
\hline \multicolumn{3}{|l|}{ Variables } & $\begin{array}{l}\text { All patients } \\
(n=133)\end{array}$ & $\begin{array}{c}\text { Male } \\
(n=83)\end{array}$ & $\begin{array}{c}\text { Female } \\
(n=50)\end{array}$ & $P$ \\
\hline \multicolumn{3}{|l|}{ Age (yr) } & $63.9 \pm 7.0$ & $64.3 \pm 6.8$ & $63.3 \pm 7.4$ & NS \\
\hline \multicolumn{3}{|c|}{ Duration after gastrectomy (yr) } & $2.7 \pm 2.4$ & $2.8 \pm 2.6$ & $2.5 \pm 2.2$ & NS \\
\hline \multicolumn{3}{|l|}{ BMI $\left(\mathrm{kg} / \mathrm{m}^{2}\right)$} & $21.5 \pm 3.3$ & $21.7 \pm 3.1$ & $21.2 \pm 3.7$ & NS \\
\hline \multicolumn{3}{|c|}{ BMI change after gastrectomy $\left(\mathrm{kg} / \mathrm{m}^{2}\right)$} & $-2.2 \pm 2.4$ & $-2.0 \pm 2.4$ & $-2.7 \pm 2.3$ & $<0.05$ \\
\hline \multicolumn{3}{|l|}{$\operatorname{AGC/EGC~}(n)$} & $75 / 58$ & $42 / 41$ & $33 / 17$ & NS \\
\hline \multirow{4}{*}{\multicolumn{2}{|c|}{ TNM stage }} & 1 & 76 & 56 & 20 & $<0.05$ \\
\hline & & 2 & 26 & 15 & 11 & \\
\hline & & 3 & 25 & 10 & 15 & \\
\hline & & 4 & 6 & 2 & 4 & \\
\hline \multirow[t]{3}{*}{ Operation type } & Subtotal gastrectomy & Billroth I & 47 & 30 & 17 & NS \\
\hline & & Billroth II & 54 & 34 & 20 & \\
\hline & Total gastrectomy & Roux - en - $Y$ & 32 & 19 & 13 & \\
\hline \multicolumn{3}{|c|}{ Bone pain } & 61 & 39 & 22 & NS \\
\hline \multicolumn{3}{|c|}{ BMI $<18.5\left(\mathrm{~kg} / \mathrm{m}^{2}\right)$} & 21 & 10 & 11 & 0.07 \\
\hline \multicolumn{3}{|c|}{ Family history of osteoporosis } & 10 & 6 & 4 & NS \\
\hline \multicolumn{3}{|l|}{ Type 2 diabetes } & 13 & 8 & 5 & NS \\
\hline \multicolumn{3}{|l|}{ Anemia } & 49 & 20 & 29 & $<0.01$ \\
\hline \multicolumn{3}{|l|}{$\mathrm{sALP}>127 \mathrm{U} / \mathrm{L}$} & 18 & 12 & 6 & NS \\
\hline \multicolumn{3}{|c|}{ Past history of fracture } & 24 & 14 & 10 & NS \\
\hline \multicolumn{3}{|c|}{ Vertebral deformity } & 62 & 36 & 26 & NS \\
\hline
\end{tabular}

AGC: Advanced gastric cancer; EGC: Early gastric cancer; Anemia, hemoglobin $<11 \mathrm{~g} / \mathrm{dL}$ in females, $<13 \mathrm{~g} / \mathrm{dL}$ in males.

Table 2 BMD of 133 patients with gastric adenocarcinoma patients according to sex (mean \pm SD)

\begin{tabular}{|c|c|c|c|c|c|c|}
\hline & \multicolumn{3}{|c|}{ Male } & \multicolumn{3}{|c|}{ Female } \\
\hline & BMD & T score & Z score & BMD & T score & Z score \\
\hline L1-4 & $0.979 \pm 0.203$ & $-1.65 \pm 1.63$ & $-0.55 \pm 1.55$ & $0.835 \pm 0.243$ & $-2.31 \pm 1.94$ & $-0.72 \pm 1.49$ \\
\hline Femur - neck & $0.837 \pm 0.145$ & $-0.89 \pm 1.13$ & $0.44 \pm 0.97$ & $0.765 \pm 0.170$ & $-1.11 \pm 1.41$ & $0.74 \pm 1.14$ \\
\hline Ward's & $0.658 \pm 0.151$ & $-1.72 \pm 1.17$ & $0.04 \pm 1.01$ & $0.588 \pm 0.181$ & $-2.26 \pm 1.40$ & $0.13 \pm 1.13$ \\
\hline Trochanter & $0.768 \pm 0.154$ & $-0.12 \pm 1.40$ & $0.65 \pm 1.28$ & $0.622 \pm 0.139$ & $-1.20 \pm 1.24$ & $-0.07 \pm 0.96$ \\
\hline Total hip & $0.911 \pm 0.181$ & $-0.25 \pm 1.45$ & $0.79 \pm 1.32$ & $0.803 \pm 0.174$ & $-1.12 \pm 1.44$ & $0.37 \pm 1.13$ \\
\hline
\end{tabular}

T scores (compared with young adults); Z scores (compared with age-matched controls).

(odds ratio, 3.923; 95\% CI, 1.202-12.805; $P=0.018$ ) in male patients, and back pain (odds ratio, 10.272; 95\% CI, 2.297-45.942; $P=0.001)$ in female patients (Table 4).

\section{DISCUSSION}

In the present study, we examined BMD in gastric adenocarcinoma patients who underwent curative surgery and analyzed the factors that were related to osteoporosis. Hemoglobin level in men and back pain in women were independent risk factors of osteoporosis by multivariate analysis.

The overall prevalence of osteoporosis in gastric adenocarcinoma patients aged $>50$ years was $39.6 \%$. The osteoporosis rate of lumbar spine BMD was $29.8 \%$ in male and $54.5 \%$ in female patients. However, the osteoporosis rate of femur neck BMD was $11.9 \%$ in male and $26.3 \%$ in female patients. This difference might be explained by the fact that the $\mathrm{Z}$ score of lumbar spine BMD was significantly lower than that of femur neck BMD. It is consistent with other studies that trabecular bone was more affected than cortical bone after gastrectomy ${ }^{[7,8]}$. In other DXA-based studies in post-gastrectomy patients, the prevalence of spinal osteoporosis was $22 \%-37 \%$ and the prevalence of femoral neck osteoporosis was $10 \%-61 \%{ }^{[9-11]}$. In the present study, 24 patients $(18.0 \%)$ reported osteoporotic fracture with severe pain, such as Colles', ankle and severe vertebral fractures. Sixty-one (45.9\%) patients complained of back pain and $62(46.6 \%)$ showed vertebral bone deformity. Thirty-three $(24.8 \%)$ patients complained of persistent back pain with vertebral deformity. Although it is not clear whether vertebral deformity with back pain directly indicates vertebral fracture, Zittel et al have reported that the overall vertebral fracture rate after gastrectomy was $31 \%$ by CT ${ }^{[12]}$. Recently, Gallacher et al have reported that vertebral deformity was found in $47 \%$ of osteoporosis patients and $23 \%$ of normal subjects $^{[23]}$. In our study, we found vertebral deformity in $64.7 \%$ of osteoporosis patients and $22.5 \%$ of those with normal BMD.

The prevalence of osteoporosis was high in females, as previously reported ${ }^{[5,11,13]}$. However, this was mainly due to the effect of $\operatorname{sex}^{[24]}$. When we compared the $\mathrm{Z}$ scores of each sex, there was no difference in the decreased proportion of BMD, as in previous reports ${ }^{[14]}$. With the same age, women showed a greater osteoporosis rate than men. Except for estrogen deficiency, a high rate of anemia and low BMI might play some role in osteoporosis. Tovey 

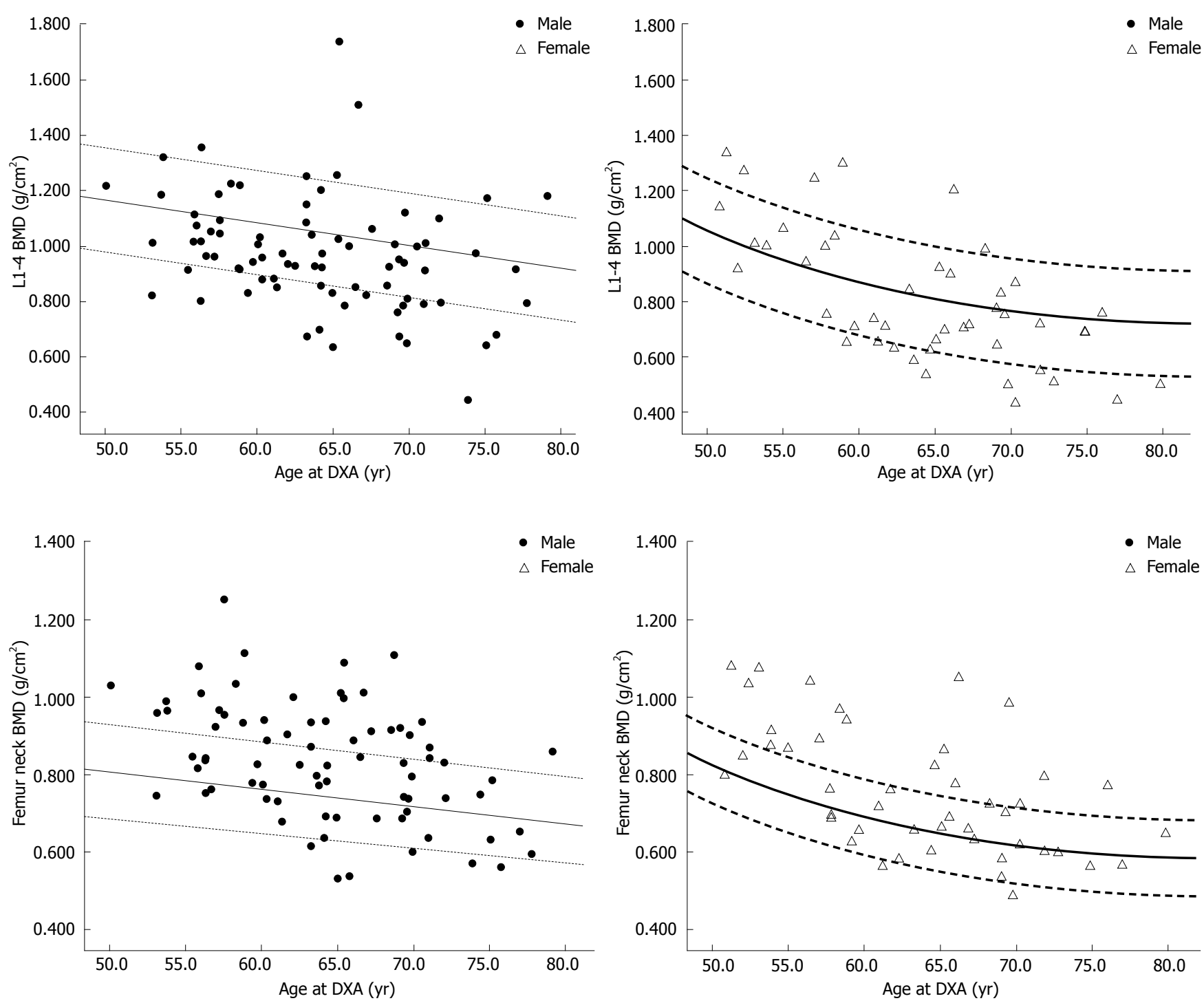

Figure $1 \mathrm{BMD}$ of lumbar spine and femur neck. Solid line represents $Z$ score $=0$. Dashed line represents $Z$ score $=-1.0$.

et al have also reported that iron deficiency anemia and osteoporosis are higher in female than in male patients throughout follow up after gastrectomy ${ }^{[5]}$.

In the present study, there was no difference between BMD or osteoporosis rates between early and advanced gastric cancer. There was no difference in osteoporosis rates according to TNM stage. In addition, there was no difference in BMD and osteoporosis rate among patients undergoing Billroth I, Billroth II and Rouxen-Y reconstruction. Although some studies have shown that Billroth II reconstruction patients have lower BMD compared to those undergoing Billroth I reconstruction ${ }^{[15,16]}$, our results showed no difference in osteoporosis rate in relation to surgical reconstruction type, as in previous studies ${ }^{[12,17]}$. These results indicate that gastrectomy itself affects BMD rather than the reconstruction method. The osteoporosis rate in gastrectomy patients cannot be predicted by reconstruction type, at least in surviving patients.

Anemia was a significant predictor for osteoporosis, at least in male patients. In multiple regression analysis, hemoglobin level was correlated with BMD in male patients $(P<0.05)$. In multivariate analysis, anemia was an independent predictor of osteoporosis (odds ratio, 4.887; $P=0.011)$. After gastrectomy and reconstruction, gastrointestinal physiology might be altered, and some nutrients might bypass the normal intestinal surface ${ }^{[6]}$. The main sites affected after gastrectomy are the duodenum and proximal jejunum, which are the main sites of calcium and iron absorption. In this study, we could not find an association between anemia and osteoporosis in females, as the rates were too high. The other factors such as age and BMI played a more important role in female patients. In females, $19(86.4 \%)$ out of 22 patients who complained of back pain had osteoporosis. It is well known that new vertebral fractures are associated with substantial increases in back pain and functional limitation in older women ${ }^{[25]}$.

This study had some limitations. We excluded patients who had been diagnosed as having previous osteoporosis. Therefore, selection bias might exist in many older patients and those who survived for long periods after gastrectomy. Although the postoperative duration or change in BMI did not show any impact on BMD or osteoporosis rate in our study, as in previous studies ${ }^{[17,18]}$, some have reported 


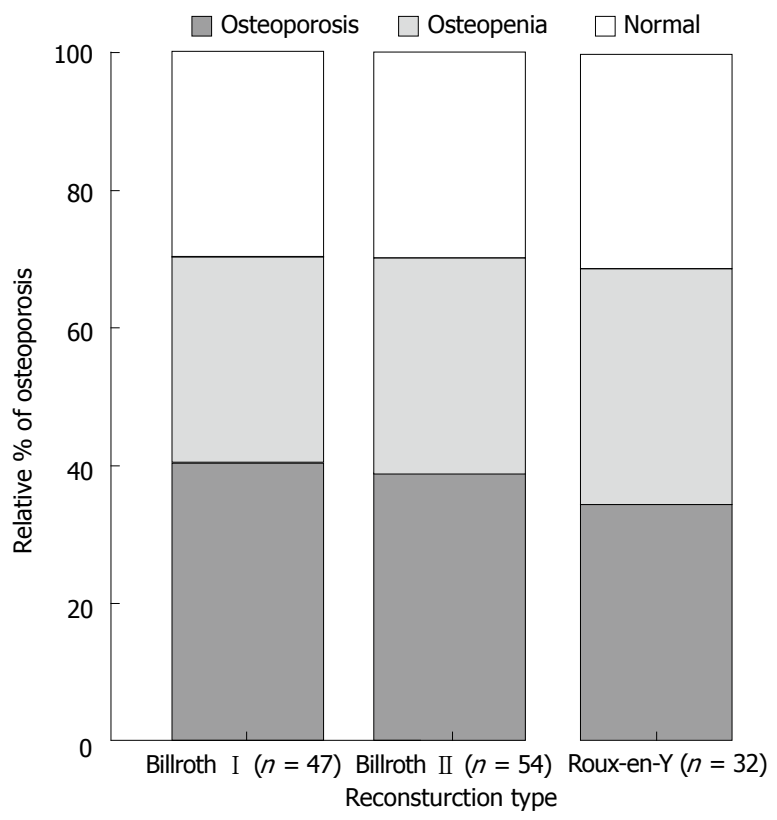

Figure 2 There was no difference in relative percentage of osteoporosis, osteopenia and normal BMD according to type of reconstruction in post-gastrectomy patients.

a negative correlation between BMD and the time interval after gastrectomy ${ }^{[14,19]}$. The other limitation was that 18 $(13.5 \%)$ patients showed increased sALP $\geqslant 127 \mathrm{U} / \mathrm{L}$. In these cases, we could not rule out osteomalacia. Others have reported that $10 \%-20 \%$ of patients after gastrectomy might have osteomalacia ${ }^{[20,26]}$.

In summary, we demonstrated high prevalence of osteoporosis and vertebral bone deformity in gastric cancer patients who underwent gastrectomy. Many also complained of back pain and a previous history of fractures. Considering the fact that gastric cancer patients after gastrectomy have many risk factors for osteoporosis fracture ${ }^{[27]}$, early diagnosis and treatment are necessary. Anemia might be an important predictive factor of osteoporosis in the clinical setting. Although some early results suggest that post-gastrectomy osteoporosis is resistant to treatment ${ }^{[5]}$, effective results have recently been reported with the development of many anti-osteoporosis drugs $^{[28]}$.

\section{COMMENTS}

\section{Background}

Post-gastrectomy bone disease may arise secondary to total or partial gastrectomy. The exact nature of the bone defect is unknown, although both osteoporosis and osteomalacia have been found. The incidence of osteoporosis is between $32 \%$ and $42 \%$. These patients are associated with an increased risk of fracture. There is no difference in risk for post-gastrectomy bone disease between those undergoing a Billroth I and II procedure.

\section{Research frontiers}

Recent studies have confirmed normal intestinal absorption of vitamin $D$ in postgastrectomy patients, although patients with post-gastrectomy steatorrhea have abnormal vitamin $\mathrm{D}$ absorption. Post-gastrectomy patients may alter their diet, and reduced serum 25-hydroxy-vitamin D level may in part reflect reduced dietary intake of vitamin $D$. Serum calcium and phosphate levels are most often normal post-gastrectomy, although calcium levels may be normal as a result of mobilization of calcium from bone. SALP, vitamin D metabolites and parathyroid
Table 3 Univariate analysis for factors associated with osteoporosis

\begin{tabular}{lcc}
\hline Variables & Patient number & $\boldsymbol{P}$ \\
\hline Age $(\mathrm{yr})<64 / \geqslant 64$ & $65 / 68$ & 0.001 \\
Male/female & $83 / 50$ & 0.006 \\
Duration after gastrectomy $(\mathrm{yr})<3 / \geqslant 3$ & $88 / 45$ & 0.573 \\
AGC/EGC & $75 / 58$ & 0.858 \\
TNM stage I / II / III /IV & $76 / 26 / 25 / 6$ & 0.164 \\
Total/partial gastrectomy & $31 / 102$ & 0.528 \\
Operation type Billroth I / II / Roux-en Y & $47 / 54 / 32$ & 0.858 \\
BMI $<18.5 / \geqslant 18.5 \mathrm{~kg} / \mathrm{m}^{2}$ & $21 / 112$ & 0.001 \\
Anemia; males, Hb $<13 / \geqslant 13$; females, Hb $<11 / \geqslant 11$ & $49 / 84$ & 0.001 \\
sALP $<127 / \geqslant 127 \mathrm{U} / \mathrm{L}$ & $115 / 18$ & 0.040 \\
Previous fracture history +/- & $24 / 109$ & 0.360 \\
Family history of osteoporosis $+/-$ & $8 / 125$ & 0.482 \\
Type 2 diabetes +/- & $13 / 111$ & 0.369 \\
Back pain $+/-$ & $61 / 72$ & 0.001 \\
Vertebral deformity +/- & $62 / 71$ & 0.001 \\
\hline
\end{tabular}

Hb: Hemoglobin.

Table 4 Multivariate analysis for factors associated with osteoporosis after stratification by sex

\begin{tabular}{lllc}
\hline & Variables & Odds ratio $\mathbf{( 9 5 \%} \mathbf{C I})$ & $\boldsymbol{P}$ \\
\hline \multirow{2}{*}{ Male } & Anemia & $4.887(1.404-17.011)$ & 0.011 \\
& Age $\geqslant 64 \mathrm{yr}$ & $3.923(1.202-12.805)$ & 0.018 \\
\multirow{2}{*}{ Female } & Back pain & $10.272(2.297-45.942)$ & 0.001 \\
\hline
\end{tabular}

hormone levels are variable post-gastrectomy. Patients who are at least 10 years post-gastrectomy (especially postmenopausal women, men aged $>50$ years, and patients with low-trauma fractures), should undergo DXA testing.

\section{Innovations and breakthroughs}

This study confirms previous observations that the prevalence of osteoporosis is high in patients following gastrectomy. As noted previously, there is no difference in $\mathrm{BMD}$ and osteoporosis rate among patients undergoing Billroth I, Billroth II and Roux-en-Y reconstruction. A number of variables have been assessed in an attempt to identify factors that predispose to reduced BMD post-gastrectomy. The authors identified that hemoglobin level and age in men and back pain in women were independent risk factors of osteoporosis, by multivariate analysis. Increased time from surgery did not correlate with lower BMD.

\section{Peer review}

This is an interesting paper from South Korea. The data presented are clear and concise and have been described clearly in the text.

\section{REFERENCES}

1 Parkin DM, Bray F, Ferlay J, Pisani P. Global cancer statistics, 2002. CA Cancer J Clin 2005; 55: 74-108

2 Lee HJ, Yang HK, Ahn YO. Gastric cancer in Korea. Gastric Cancer 2002; 5: 177-182

3 Iba K, Wada T, Takada J, Yamashita T. Multiple insufficiency fractures with severe osteoporosis. J Orthop Sci 2003; 8: 717-720

4 Liedman B. Symptoms after total gastrectomy on food intake, body composition, bone metabolism, and quality of life in gastric cancer patients--is reconstruction with a reservoir worthwhile? Nutrition 1999; 15: 677-682

5 Tovey FI, Hall ML, Ell PJ, Hobsley M. A review of postgastrectomy bone disease. J Gastroenterol Hepatol 1992; 7: 639-645

6 Bernstein CN, Leslie WD, Leboff MS. AGA technical review on osteoporosis in gastrointestinal diseases. Gastroenterology 2003; 124: 795-841 
$7 \quad$ Klein KB, Orwoll ES, Lieberman DA, Meier DE, McClung MR, Parfitt AM. Metabolic bone disease in asymptomatic men after partial gastrectomy with Billroth II anastomosis. Gastroenterology 1987; 92: 608-616

8 Resch H, Pietschmann P, Pernecker B, Krexner E, Willvonseder $R$. The influence of partial gastrectomy on biochemical parameters of bone metabolism and bone density. Clin Investig 1992; 70: 426-429

9 Liedman B, Bosaeus I, Mellström D, Lundell L. Osteoporosis after total gastrectomy. Results of a prospective, clinical study. Scand J Gastroenterol 1997; 32: 1090-1095

10 Schmiedl A, Schwille PO, Stühler C, Göhl J, Rümenapf G. Low bone mineral density after total gastrectomy in males: a preliminary report emphasizing the possible significance of urinary net acid excretion, serum gastrin and phosphorus. Clin Chem Lab Med 1999; 37: 739-744

11 Adachi Y, Shiota E, Matsumata T, Iso Y, Yoh R, Kitano S. Osteoporosis after gastrectomy: bone mineral density of lumbar spine assessed by dual-energy X-ray absorptiometry. Calcif Tissue Int 2000; 66: 119-122

12 Zittel TT, Zeeb B, Maier GW, Kaiser GW, Zwirner M, Liebich H, Starlinger M, Becker HD. High prevalence of bone disorders after gastrectomy. Am J Surg 1997; 174: 431-438

13 Nishimura O, Furumoto T, Nosaka K, Kouno K, Sumikawa M, Hisaki T, Odachi T, Mizumoto K, Kishimoto H, Yamamoto $\mathrm{K}$. Bone disorder following partial and total gastrectomy with reference to bone mineral content. Jpn J Surg 1986; 16: 98-105

14 Nihei Z, Kojima K, Ichikawa W, Hirayama R, Mishima Y. Chronological changes in bone mineral content following gastrectomy. Surg Today 1996; 26: 95-100

15 Filipponi P, Gregorio F, Cristallini S, Mannarelli C, Blass A, Scarponi AM, Vespasiani G. Partial gastrectomy and mineral metabolism: effects on gastrin-calcitonin release. Bone Miner 1990; 11: 199-208

16 Mellström D, Johansson C, Johnell O, Lindstedt G, Lundberg PA, Obrant K, Schöön IM, Toss G, Ytterberg BO. Osteoporosis, metabolic aberrations, and increased risk for vertebral fractures after partial gastrectomy. Calcif Tissue Int 1993; 53: 370-377

17 Inoue K, Shiomi K, Higashide S, Kan N, Nio Y, Tobe T, Shigeno C, Konishi J, Okumura H, Yamamuro T. Metabolic bone disease following gastrectomy: assessment by dual energy X-ray absorptiometry. Br J Surg 1992; 79: 321-324

18 Heiskanen JT, Kröger H, Pääkkönen M, Parviainen MT,
Lamberg-Allardt C, Alhava E. Bone mineral metabolism after total gastrectomy. Bone 2001; 28: 123-127

19 Imamura M, Yamauchi H, Fukushima K, Sasaki I, Ouchi A. Bone metabolism following gastric surgery: microdensitometry and single-photon absorptiometry. Tohoku J Exp Med 1988; 156: 237-249

20 Eddy RL. Metabolic bone disease after gastrectomy. Am J Med 1971; 50: 442-449

21 Genant HK, Cooper C, Poor G, Reid I, Ehrlich G, Kanis J, Nordin BE, Barrett-Connor E, Black D, Bonjour JP, DawsonHughes B, Delmas PD, Dequeker J, Ragi Eis S, Gennari C, Johnell O, Johnston CC Jr, Lau EM, Liberman UA, Lindsay R, Martin TJ, Masri B, Mautalen CA, Meunier PJ, Khaltaev $\mathrm{N}$. Interim report and recommendations of the World Health Organization Task-Force for Osteoporosis. Osteoporos Int 1999; 10: $259-264$

22 Genant HK, Wu CY, van Kuijk C, Nevitt MC. Vertebral fracture assessment using a semiquantitative technique. J Bone Miner Res 1993; 8: 1137-1148

23 Gallacher SJ, Gallagher AP, McQuillian C, Mitchell PJ, Dixon $\mathrm{T}$. The prevalence of vertebral fracture amongst patients presenting with non-vertebral fractures. Osteoporos Int 2007; 18: $185-192$

24 Dennison E, Cooper C. Epidemiology of osteoporotic fractures. Horm Res 2000; 54 Suppl 1: 58-63

25 Nevitt MC, Ettinger B, Black DM, Stone K, Jamal SA, Ensrud K, Segal M, Genant HK, Cummings SR. The association of radiographically detected vertebral fractures with back pain and function: a prospective study. Ann Intern Med 1998; 128: 793-800

26 Bisballe S, Eriksen EF, Melsen F, Mosekilde L, Sørensen $\mathrm{OH}$, Hessov I. Osteopenia and osteomalacia after gastrectomy: interrelations between biochemical markers of bone remodelling, vitamin D metabolites, and bone histomorphometry. Gut 1991; 32: 1303-1307

27 Brown JP, Josse RG. 2002 clinical practice guidelines for the diagnosis and management of osteoporosis in Canada. CMAJ 2002; 167: S1-S34

28 Suzuki Y, Ishibashi Y, Omura N, Kawasaki N, Kashiwagi H, Yanaga K, Abo M, Urashima M. Alendronate improves vitamin D-resistant osteopenia triggered by gastrectomy in patients with gastric cancer followed long term. J Gastrointest Surg 2005; 9: 955-960

S- Editor Dhiman RK L- Editor Kerr C E- Editor Lu W 